\title{
Structural Diversity and Biological Activities of Diterpenoids Derived from Euphorbia fischeriana Steud
}

\author{
Baiyu Jian ${ }^{1}$, Hao Zhang ${ }^{2}$ and Jicheng Liu ${ }^{2, *}$ \\ 1 Graduate School of Heilongjiang University of Chinese Medicine, \\ Heilongiiang University of Chinese Medicine, Harbin 150040, China; abcaaa1@126.com \\ 2 Research Institute of Medicine and Pharmacy, Qiqihar Medical University, Qiqihar 161006, China; \\ zhang101hao@aliyun.com \\ * Correspondence: Jcliu@qmu.edu.cn; Tel.: +86-452-266-3333
}

Received: 9 March 2018; Accepted: 6 April 2018; Published: 18 April 2018

\begin{abstract}
Diterpenoids are the focus of natural product drug discovery because of their great structural diversity and pronounced biological activities. Euphorbia fischeriana Steud is a Chinese traditional medicinal herb for curing edema, ascites, and cancer. This plant contains rich diterpenoids. Based on the carbon skeleton and substituents, it can be classified into thirteen subtypes: ent-abietane, daphnane, tigliane, ingenane, ent-atisane, ent-rosane, ent-kaurene, ent-kaurane, secotigliane, lathyrane, ent-pimarene, isopimarene and dimeric. In this paper, we reviewed the chemical structures and biological activities of 90 diterpenoids isolated from this medicinal herb. We hope that this work can serve as a reference for further research of these diterpenoids and lay the foundation for drug discovery.
\end{abstract}

Keywords: diterpenoids; Euphorbia fischeriana Steud; bioactivity

\section{Introduction}

Euphorbia fischeriana Steud is a perennial herbaceous plant belonging to the family Euphorbiaceae, which is primarily distributed in northeastern mainland China [1]. The dried plant roots of E. fischeriana in traditional Chinese medicine, have been used as a remedy for the treatment of ailments, including edema, ascites and cancer [2-4]. The main components of E. fischeriana are diterpenoids, triterpenes, steroids, aromatic compounds and tannins [3]. Among them, diterpenoids are the primary bioactive constituents of this plant. Diterpenoids are a focus of natural product drug discovery because of their great structural diversity and pronounced biological activities [5]. In the last few years, a tigliane-type diterpenoid prostratin extracted from the roots of E. fischeriana has attracted great interest because of its unique ability to activate latent viral reservoirs and protect healthy cells from infection $[5,6]$. It is a protein kinase $\mathrm{C}$ activator that can reactivate HIV-1 latency and help to avoid the new infection of CD4+ Cells [5,6]. It brings new hope for the treatment of HIV. This result encouraged us to screen more bioactive diterpenoids from $E$. fischeriana for disease treatment.

In this review article, we focus on the structures and biological activities of 90 diterpenoids with different structures derived from E. fischeriana. We hope this work can serve as a reference for drug discovery.

\section{Chemical Structure of Diterpenoids Derived from E. fischeriana}

Diterpenoids are the major components of E. fischeriana. According to the literature, almost 90 diterpenoids have been isolated from the roots of this plant since the 1970s. Types of 
diterpenoids include ent-abietane, daphnane, tigliane, ingenane, ent-atisane, ent-rosane, ent-kaurene, ent-kaurane, secotigliane lathyrane, ent-pimarene, isopimarene and dimeric. Their names, subtypes and corresponding references are compiled in Table 1. Their chemical structures (1-90) are shown in Figure 1.

Table 1. Emerging diterpenoids in E. fischeriana.

\begin{tabular}{|c|c|c|c|}
\hline No. & Compound & Subtype & Ref. \\
\hline 1 & jolkinolide B & ent-abietane & [3] \\
\hline 2 & jolkinolide A & ent-abietane & [3] \\
\hline 3 & 17-hydroxyjolkinolide B & ent-abietane & [3] \\
\hline 4 & 17-acetoxyjolkinolide B & ent-abietane & [3] \\
\hline 5 & 17-hydroxyjolkinolide A & ent-abietane & [3] \\
\hline 6 & $13 \beta$-hydroxy-ent-abiet-8(14)-en-7-one & ent-abietane & [6] \\
\hline 7 & 17-acetoxyjolkinolide A & ent-abietane & [2] \\
\hline 8 & 11ß-hydroxy-8,14-epoxy-ent-abieta-13(15)-en-16,12-olide & ent-abietane & [2] \\
\hline 9 & euphorin E & ent-abietane & [7] \\
\hline 10 & euphorin F & ent-abietane & [7] \\
\hline 11 & euphorin G & ent-abietane & [7] \\
\hline 12 & euphorin $\mathrm{H}$ & ent-abietane & [7] \\
\hline 13 & ent-11 $\alpha$-hydroxy-abieta-8(14),13(15)-dien-16,12 $\alpha$-olide & ent-abietane & {$[7]$} \\
\hline 14 & $11 \alpha, 17$-dihydroxyhelioscopinolide E & ent-abietane & [1] \\
\hline 15 & $6 \beta, 11 \alpha, 17$-trihydroxyhelioscopinolide $\mathrm{E}$ & ent-abietane & {$[1]$} \\
\hline 16 & 11-oxo-ebracteolatanolide B & ent-abietane & [1] \\
\hline 17 & 7-deoxylangduin B. & ent-abietane & [1] \\
\hline 18 & ent-11 $\beta$-hydroxyabieta- $8(14), 13(15)$-dien- $16,12 \beta$-olide & ent-abietane & [2] \\
\hline 19 & $7 \beta, 11 \beta, 12 \beta$-trihydroxy-ent-abieta- $8(14), 13$ (15)-dien-16,12-olide & ent-abietane & {$[1]$} \\
\hline 20 & langduin B & ent-abietane & [1] \\
\hline 21 & $\begin{array}{l}\text { (4R,4aR)-dihydroxy-3-hydroxymethyl-7,7,10a-trimethyl- } \\
2,4,4 a, 5,6,6 a, 7,8,9,10,10 a, 10 b \text {-dodecahydrophenanthro[3,2-b]furan-2-one }\end{array}$ & ent-abietane & [1] \\
\hline 22 & yuexiandajisu E & ent-abietane & [1] \\
\hline 23 & yuexiandajisu D & ent-abietane & {$[8,9]$} \\
\hline 24 & fischeriolide A & entt-abietane & [8] \\
\hline 25 & fischeriolide B & ent-abietane & {$[8]$} \\
\hline 26 & fischeriolide $\mathrm{C}$ & entt-abietane & {$[8]$} \\
\hline 27 & fischeriolide D & ent-abietane & [8] \\
\hline 28 & prostratin & tigliane & {$[3]$} \\
\hline 29 & 14-didehydrolangduin A & tigliane & [10] \\
\hline 30 & langduin $\mathrm{F}$ & tigliane & [10] \\
\hline 31 & 3-hydroxyl-4-dehydro-10-dehydroxylphorbol & tigliane & [10] \\
\hline 32 & 12-deoxyphorbol 13-palmitate & tigliane & [3] \\
\hline 33 & 12-deoxyphorbaldehyde-13-hexadecacetate & tigliane & {$[3]$} \\
\hline 34 & 12-deoxyphorbol 13-(9Z)-octadecenoate 20-acetate & tigliane & {$[4,10]$} \\
\hline 35 & 13-O-acetyl-phorbol & tigliane & [11] \\
\hline 36 & 12-deoxyphorbaldehyde-13-acetate & tigliane & [3] \\
\hline 37 & fischeroside A & tigliane & [12] \\
\hline 38 & fischeroside B & tigliane & [12] \\
\hline 39 & fischeroside $\mathrm{C}$ & tigliane & [12] \\
\hline 40 & 12-deoxyphorbol-13,20-diacetate & tigliane & {$[12]$} \\
\hline 41 & 9-deoxy-11 $\beta$-hydroxyprostratin & tigliane & [1] \\
\hline 42 & prostratin $20-O-\left(4^{\prime}\right.$-galloyl $)-\beta$-D-glucopyranoside & tigliane & [1] \\
\hline 43 & prostratin $20-O-\left(3^{\prime}\right.$-galloyl $)-\beta$-D-glucopyranoside & tigliane & {$[1]$} \\
\hline 44 & langduin A & daphnane & {$[3]$} \\
\hline 45 & $\begin{array}{c}4 \beta, 9 \alpha, 20 \text {-trihydroxy-13,15-secotiglia-1,6-diene-3,13-dione } \\
\text { 20-O- } \beta \text {-D-[6-galloyl]glu-copyranoside }\end{array}$ & secotigliane & [13] \\
\hline 46 & euphopiloside A & daphnane & [13] \\
\hline 47 & ent-3 $\beta$-hydroxy-rosa- $1(10), 15$-diene & ent-rosane & [7] \\
\hline 48 & ebractenoid C & ent-rosane & [7] \\
\hline 49 & yuexiandajisu $\mathrm{F}$ & ent-rosane & [7] \\
\hline 50 & euphorin A & ent-rosane & [7] \\
\hline
\end{tabular}


Table 1. Cont.

\begin{tabular}{|c|c|c|c|}
\hline No. & Compound & Subtype & Ref. \\
\hline 51 & euphorin B & ent-rosane & [7] \\
\hline 52 & ebractenoid F & ent-rosane & [7] \\
\hline 53 & euphorin C & ent-rosane & [7] \\
\hline 54 & fischeria A & ent-rosane & [7] \\
\hline 55 & euphorin D & ent-rosane & [7] \\
\hline 56 & 3,20-dihydroxy-ent-1(10), 15-rosadiene & ent-rosane & [2] \\
\hline 57 & 3,7-dihydroxy-ent-1(10), 15-rosadiene & ent-rosane & [2] \\
\hline 58 & ent- $(3 \alpha, 5 \beta, 8 \beta, 9 \alpha, 10 \beta, 12 \beta)-3$-hydroxyatis-16-en-14-one & ent-atisane & [14] \\
\hline 59 & ent-atisane- $3 \alpha, 16 \beta, 17$-triol & ent-atisane & [14] \\
\hline 60 & ent-1$\beta, 3 \beta, 16 \beta, 17$-tetrahydroxyatisane & ent-atisane & [15] \\
\hline 61 & ent-1 $\beta, 3 \alpha, 16 \beta, 17$-tetrahydroxyatisane & ent-atisane & [15] \\
\hline 62 & ent-3 $\beta, 13 S$-dihydroxy-atis-16-en-14-one & ent-atisane & [15] \\
\hline 63 & ent-16 $\alpha, 17$-dihydroxyatisan-3-one & ent-atisane & [15] \\
\hline 64 & ent-atisane- $3 \beta, 16 \alpha, 17$-triol & ent-atisane & [15] \\
\hline 65 & ent-13-hydroxyatis-16-ene-3,14-dione & ent-atisane & [2] \\
\hline 66 & ent-13S-hydroxy-16-atisene-3,14-dione & ent-atisane & [12] \\
\hline 67 & ent-13 $\alpha$-hydroxyatis-16-ene-3,14-dione & ent-atisane & [16] \\
\hline 68 & ent-3 $\beta, 13 \alpha$-dihydroxyatis-16-ene-14-one & ent-atisane & [16] \\
\hline 69 & ent-atis-16-ene-3,14-dione & ent-atisane & [16] \\
\hline 70 & ent-3 $\beta$-hydroxyatis-16-ene-14-one & ent-atisane & [16] \\
\hline 71 & ent-3 $\beta$-hydroxyatis-16-ene-2,14-dione & ent-atisane & [16] \\
\hline 72 & ent-16 $\alpha, 17$-dihydroxyatis-16-ene-3,14-dione & ent-atisane & [16] \\
\hline 73 & 19-O- $\beta$-Dglucopyranosyl-ent-atis-16-ene-3,14-dione & ent-atisane & [16] \\
\hline 74 & 19-O-(6-galloyl)- $\beta$-D-glucopyranosyl-ent-atis-16-ene-3,14-dione & ent-atisane & [16] \\
\hline 75 & ingenol & ingenane & [7] \\
\hline 76 & ingenol-3-palmitate & ingenane & [7] \\
\hline 77 & ingenol-3-myristinate & ingenane & [7] \\
\hline 78 & ingenol-20-palmitate & ingenane & [7] \\
\hline 79 & ingenol-20-myristinate & ingenane & [7] \\
\hline 80 & ingenol-6,7-epoxy-3-tetradecanoate & ingenane & [2] \\
\hline 81 & ent-kaurane-3-oxo-17 $\beta$-ol & ent-kaurane & [14] \\
\hline 82 & ent-kaur-16-en-14-ol & ent-kaurene & [2] \\
\hline 83 & 3S,16S,17-trihydroxy-2-one-ent-kaurane & ent-kaurane & [15] \\
\hline 84 & ent-16 $\alpha, 17$-dihydroxy-kauran-3-one & ent-kaurane & [7] \\
\hline 85 & $3 \alpha, 17$-dihydroxy-ent-pimara-8(14),15-diene & ent-pimarene & [3] \\
\hline 86 & isopimara- $9(11), 15$-diene-3,19-diol & isopimarene & {$[2]$} \\
\hline 87 & ent-8(14)-pimarene-12 $\beta, 15 S, 16$-triol & ent-pimarene & [13] \\
\hline 88 & jolkinol A & lathyrane & [7] \\
\hline 89 & langduin $\mathrm{C}$ & dimeric & {$[3,17]$} \\
\hline 90 & langduin D & dimeric & [16] \\
\hline
\end{tabular}

\section{Biological Activities of Diterpenoids}

Diterpenoids isolated from E. fischeriana exert many different activities, including antitumor, anti-inflammatory, anti-HIV-1, feeding deterrent and others.

\subsection{Antitumor Activity}

Many investigations have been performed on the antitumor activity of diterpenoids from E. fischeriana. Compounds with different skeletal types (e.g., abietane, ingenane, tigliane and lathyrane) proved to have moderate or strong antiproliferative effects on different human cancer cell lines $[5,15]$. Jolkinolide B (1), a typical ent-abietane-type diterpenoid isolated from E. fischeriana, was found to induce time- and dose-dependent cytotoxicity in cells derived from liver [18], breast [19,20], gastric [18], cervical cancers [18], as well as human leukemic [21,22]. The anticancer effect of Jolkinolide B are associated with various kinds of mechanisms. Jolkinolide B is capable of inducing apoptosis in breast cancer cells through inhibition of the PI3K/Akt signaling pathway $[19,20]$. It can block cell cycles at G1 in human myeloid Leukemic cell K562 [22]. Moreover, it restrains the metastasis in breast cancer MDA-MB-231 cells through suppression of $\beta_{1}$-integrin expression and phosphorylation of focal adhesion kinase (FAK) [23]. 17-Acetoxyjolkinolide B (4), has the 
potential to irreversibly inhibit I $\kappa B$ kinase and induce apoptosis of tumor cells. Nuclear factor- $\kappa B$ (NF-kB) plays an important role in tumor cell survival, growth, angiogenesis, and metastasis. 17-Acetoxyjolkinolide $\mathrm{B}$ is a novel type of anticancer drug candidate, as a NF- $\mathrm{kB}$ pathway inhibitor [24]. 17-Hydroxyjolkinolide B (5), a natural bioactive molecule, exerts its cytotoxicity against liver [18], breast 18], gastric [18], cervical [18], lung [18], ovarian [25], prostate [25], colorectal [25], cervix cancers [25]. Thorough examination proved that 17 -hydroxyjolkinolide B can inhibit signal transducers and activators of transcription 3 signaling by covalently cross-linking Janus kinases and induce apoptosis of human cancer cells [25]. 12-deoxyphorbol 13-palmitate (32), a tigliane-type diterpenoid isolated from E. fischerian is another effective antineoplastic compound. 12-Deoxyphorbol 13-palmitate was found to mediate cell growth inhibition, G2-M cell cycle arrest and apoptosis in BGC823 cells [26]. It also reportedly has the ability to inhibit VEGF induced angiogenesis via suppression of VEGFR-2-signaling pathway [27]. Three ingenol diterpenoids including ingenol 3-palmitate (76), ingenol-3-myristinate (77), ingenol 6,7-epoxy-3-tetradecanoate (80) showed significant cytotoxicity against $\mathrm{A} 549$ with $\mathrm{IC}_{50}$ value of $3.35,2.85,2.88 \mu \mathrm{g} / \mathrm{mL}$, respectively [2]. Two ent-atisane-type diterpenoids ent-1 $\beta, 3 \beta, 16 \beta, 17$-tetrahydroxyatisane (60), ent-1 $\beta, 3 \alpha, 16 \beta, 17$-tetrahydroxyatisane (61) showed inhibitory effects against MCF-7 with IC $_{50}$ levels of 23.21 and $15.42 \mathrm{mM}$ [15]. Moreover, 13 diterpenoids, including jolkinolide B (1), euphorin E (9), euphorin $H$ (12), yuexiandajisu $E$ (22), ebractenoid C (48), ebractenoid F (52), euphorinC (53), ent-3ß-hydroxyatis-16-ene-2,14-dione (71), 19-O- $\beta$-Dglucopyranosyl-ent-atis-16-ene-3,14-dione (73), ingenol-3-palmitate (76), ingenol-3-myristinate (77), ingenol-20-myristinate (79), and jolkinol A (88) showed inhibitory activity on mammosphere formation in human breast cancer MCF-7 cells at a final concentration of $10 \mu \mathrm{M}$, suggesting the potential of these bioactive diterpenoids for further investigation of the action targeting cancer stem cells $[7,16]$. The previous article provide more detailed descriptions about the anticancer mechanisms of these bioactive diterpenoids [28].

\subsection{Anti-Inflammatory}

Jolkinolide B (1) was reported to have a protective effect on LPS-induced ALI in mice [29]. It was revealed that jolkinolide B significantly inhibited LPS-induced histological alterations, lung edema, inflammatory cell infiltration, myeloperoxidase (MPO) activity [29]. At the molecular level, jolkinolide B reduced the production of TNF- $\alpha$, IL- 6 and IL-1 $\beta$. Furthermore jolkinolide $\mathrm{B}$ was shown to inhibit LPS-induced the degradation of IKB $\alpha$ and phosphorylation of NF-kB p65 and MAPK [29]. 17-Hydroxy-jolkinolide B (5) was found to a potential anti-inflammatory drug candidate. It can inhibit LPS-induced the production of PGE 2, NO, IL-6, and TNF- $\alpha$ in RAW264 cells. 17-Hydroxy-jolkinolide B has the ability to reduce the expression of COX-2, iNOS, $I L-6$, and TNF- $\alpha$ gene through the suppression of MAPK phosphorylation and NF-KB activation. In addition, it induced the HO-1 expression [30]. Five diterpenoids including jolkinolide B (1), 11ß-hydroxy-8,14-epoxy-ent-abieta-13(15)-en-16,12 $\alpha$-olide (8), yuexiandajisu D (23), ebractenoid F (52), jolkinol A (88) exhibited promising inhibitory effects on NO production in LPS-induced RAW 264.7 macrophages [8]. These compounds may be worthy of further investigation for the treatment of inflammatory diseases associated with enhanced production of NO [8]. Here, these studies are summarized in Table 2. 


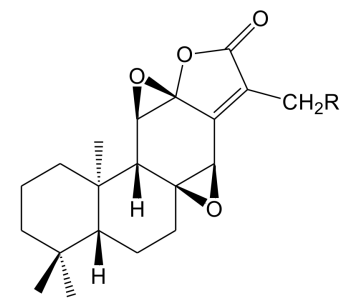

$\begin{array}{ll} & \mathrm{R} \\ 1 & \mathrm{H} \\ 3 & \mathrm{OH} \\ 4 & \mathrm{AcO}\end{array}$<smiles>CC1=C2[C@@H](O)C3=CC[C@H]4C(C)(C)CCC[C@]4(C)[C@H]3[C@H](O)[C@@H]2OC1=O</smiles>

9

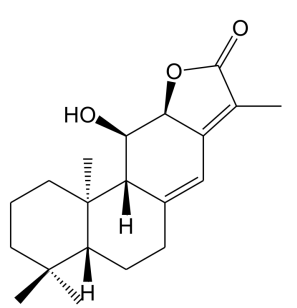

13

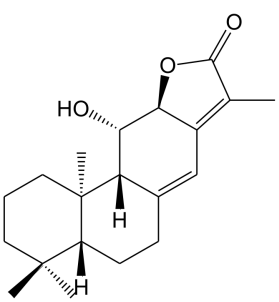

18

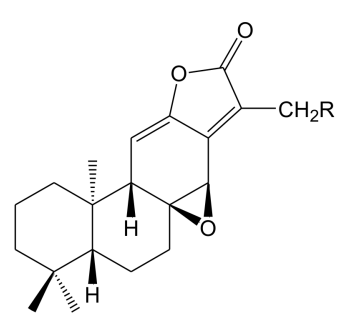

$\begin{array}{ll} & \mathrm{R} \\ 2 & \mathrm{H} \\ 5 & \mathrm{OH} \\ 7 & \mathrm{AcO}\end{array}$

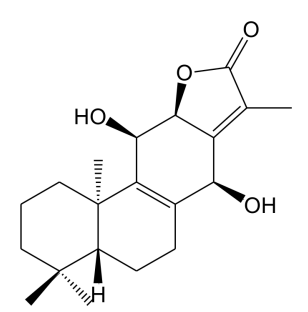

10

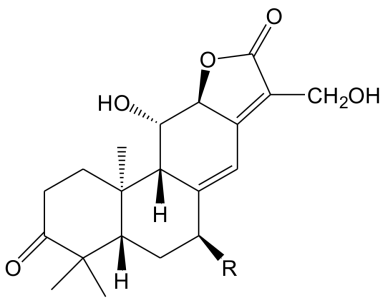

$14 \stackrel{\stackrel{R}{H}}{\mathrm{OH}}$

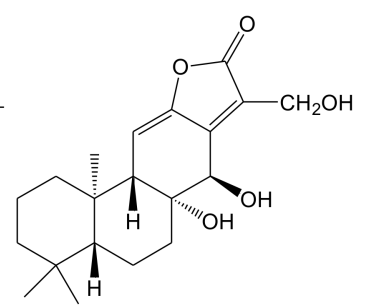

21

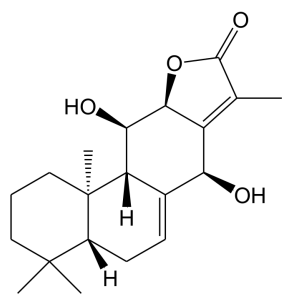

24

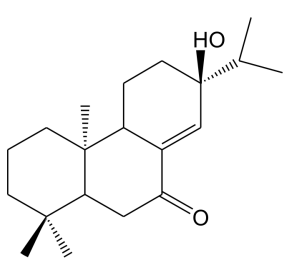

6

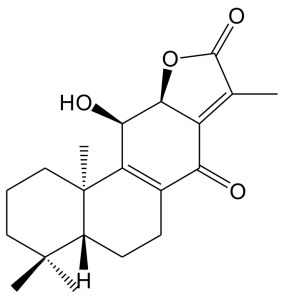

11

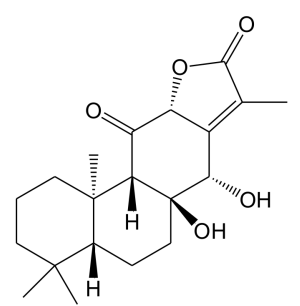

16<smiles>CC1=C2C(C)C(=O)O[C@H]2[C@@H](O)[C@@]2(O)CC[C@@H]3[C@@H](CCCC3(C)C)[C@]12C</smiles>

22

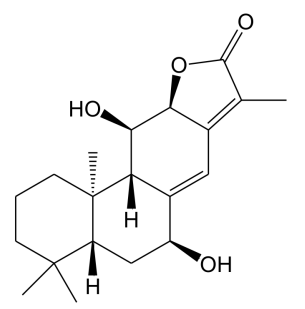

26

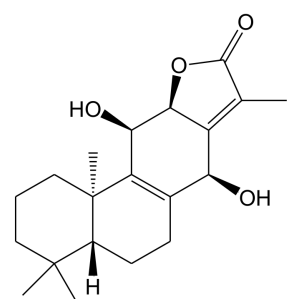

27

Figure 1. Cont. 


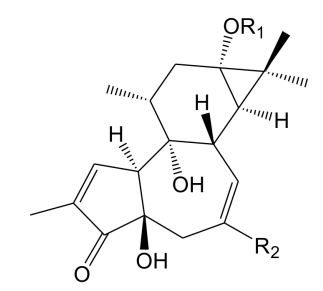

$\begin{array}{ll} & \mathrm{R}_{1} \\ 28 & \mathrm{Ac} \\ 32 & \mathrm{CH}_{3}\end{array}$

$33-\mathrm{CH}_{3}\left(\mathrm{CH}_{2}\right)_{14} \mathrm{CO}$

$33 \mathrm{CH}_{3}\left(\mathrm{CH}_{2}\right)_{14} \mathrm{CO}$

40 AC

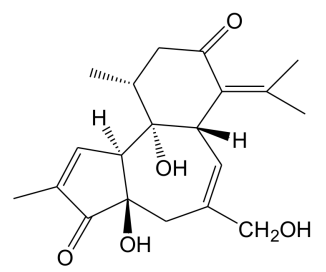

29

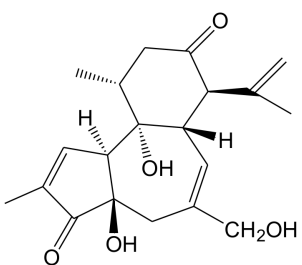

30

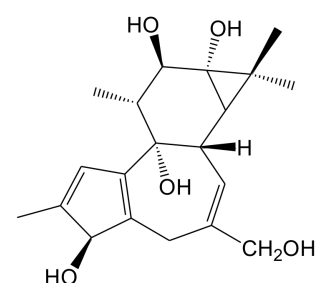

31

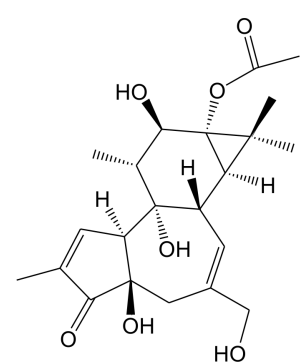

35

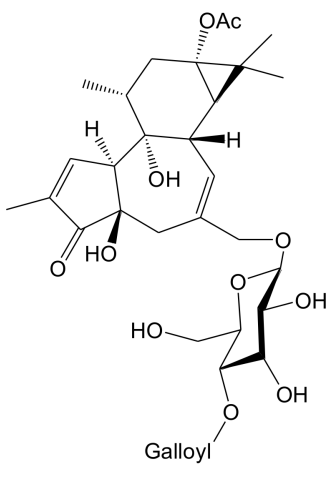

42

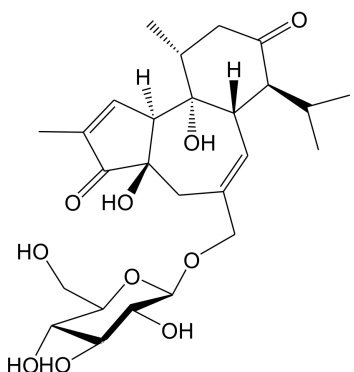

46

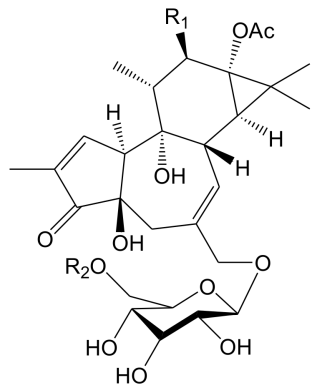

$\begin{array}{lll} & \mathrm{R}_{1} & \mathrm{R} 2 \\ 37 & \mathrm{H} & \mathrm{H}\end{array}$

$38 \mathrm{H}$ Galloyl

$39 \mathrm{OH} \mathrm{H}$

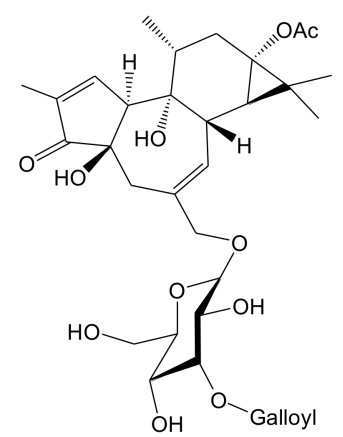

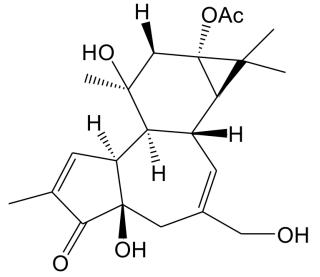

41

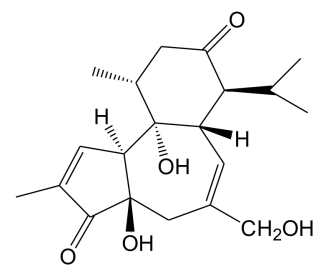

44

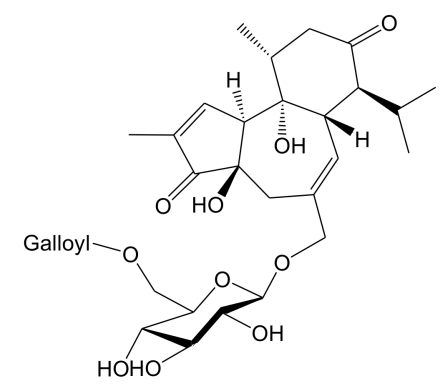

45

43

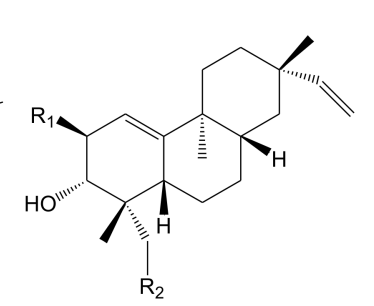

$\begin{array}{lll} & \mathrm{R}_{1} & \mathrm{R}_{2} \\ 47 & \mathrm{H} & \mathrm{H} \\ 48 & \mathrm{H} & \mathrm{OH} \\ 49 & \mathrm{OH} & \mathrm{H}\end{array}$

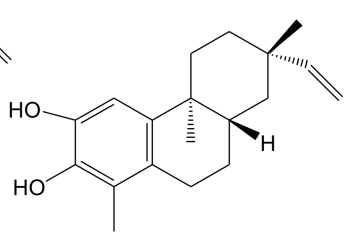

52

Figure 1. Cont. 


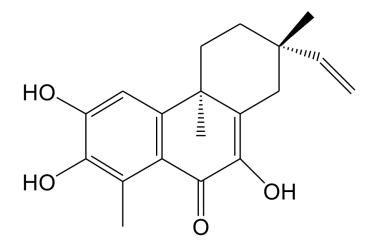

53

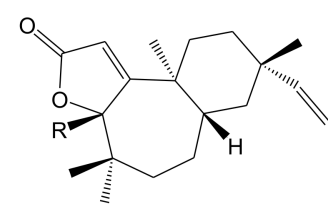

$\begin{array}{ll}\mathrm{R} \\ 55 & \stackrel{\mathrm{H}}{\mathrm{OH}}\end{array}$

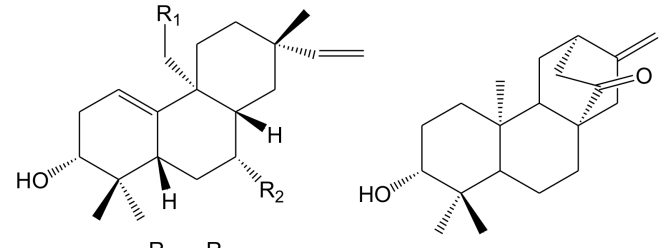

$\begin{array}{lll} & \mathrm{R}_{1} & \mathrm{R}_{2} \\ 56 & \mathrm{OH} & \mathrm{H} \\ 57 & \mathrm{H} & \mathrm{OH}\end{array}$

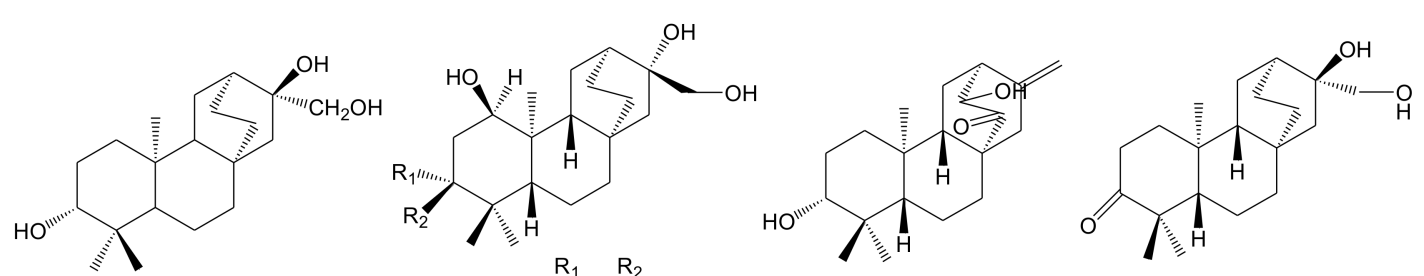

$\begin{array}{lll} & \mathrm{R}_{1} & \mathrm{R}_{2} \\ 60 & \mathrm{OH} & \mathrm{H} \\ 61 & \mathrm{H} & \mathrm{OH}\end{array}$

59

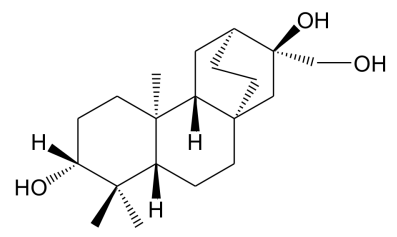

64

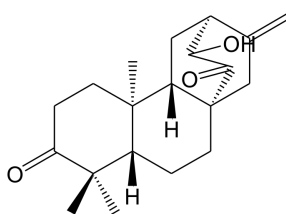

65

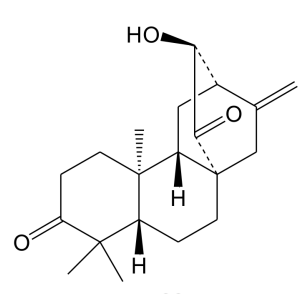

66

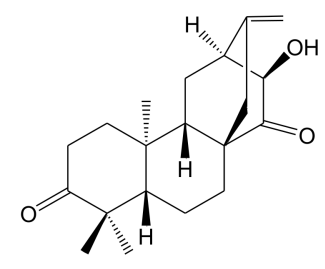

67

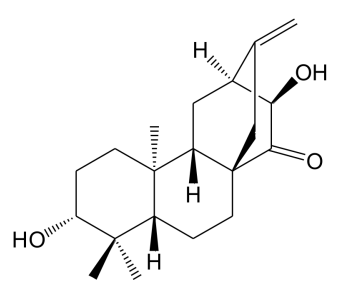

68

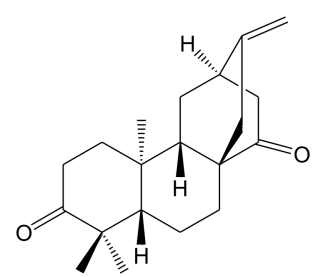

69

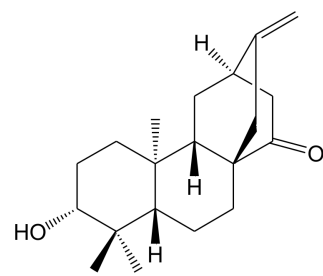

70

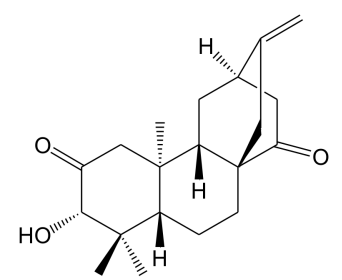

71

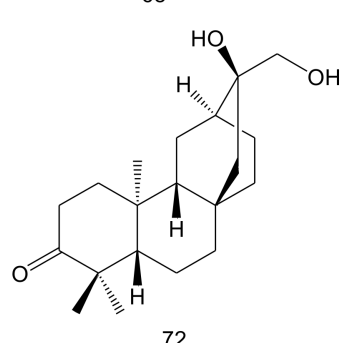

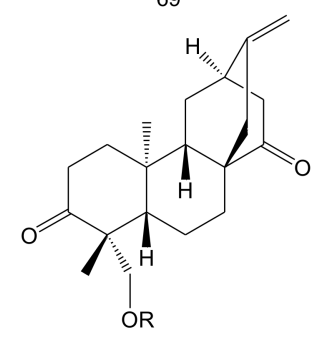

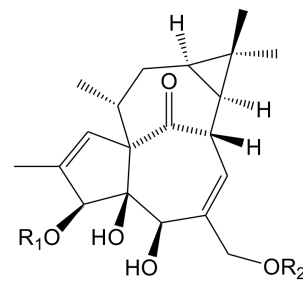

$\begin{array}{ll} & \mathrm{R}_{1} \\ 75 & \mathrm{H} \\ 76 & \mathrm{CO}\left(\mathrm{CH}_{2}\right)_{14} \mathrm{CH}_{3} \\ 77 & \mathrm{CO}\left(\mathrm{CH}_{2}\right)_{12} \mathrm{CH}_{3}\end{array}$ $\mathrm{R}_{2}$
$\mathrm{H}$
$\mathrm{H}$
$\mathrm{H}$
$\mathrm{CO}\left(\mathrm{CH}_{2}\right)_{14} \mathrm{CH}_{3}$
$\mathrm{CO}\left(\mathrm{CH}_{2}\right)_{12} \mathrm{CH}_{3}$ $\mathrm{R}$
$\beta$-D-glucopyranosyl 74 6-galloyl- $\beta$-D-glucopyranosyl

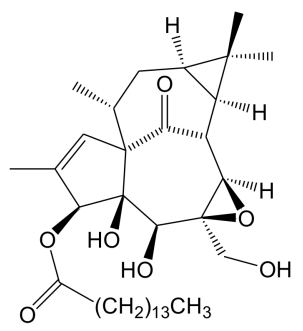

80

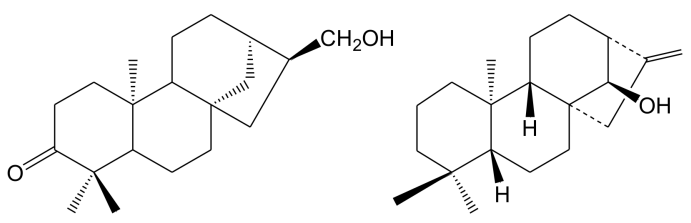

81

82

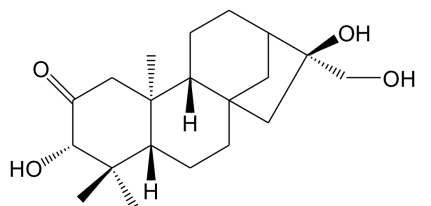

83

Figure 1. Cont. 


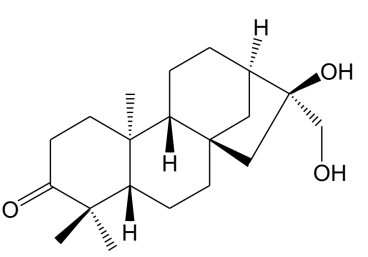

84

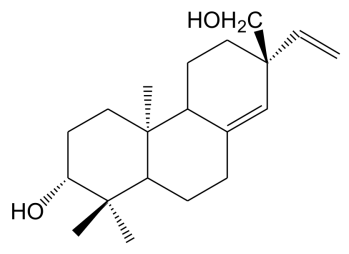

85

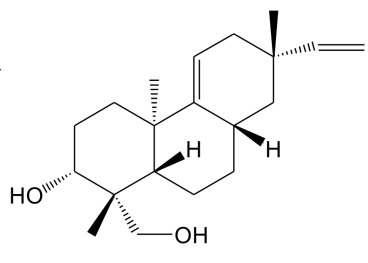

86

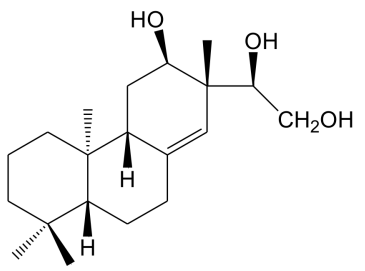

87

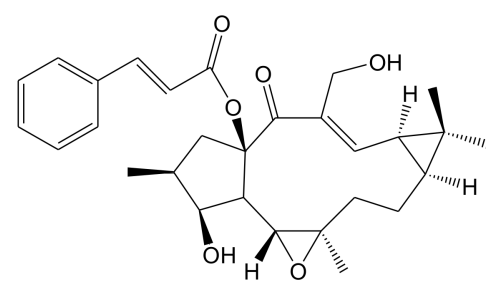

88

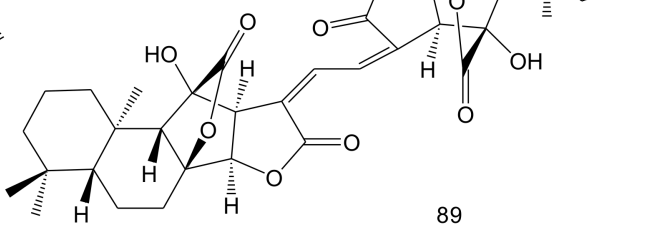

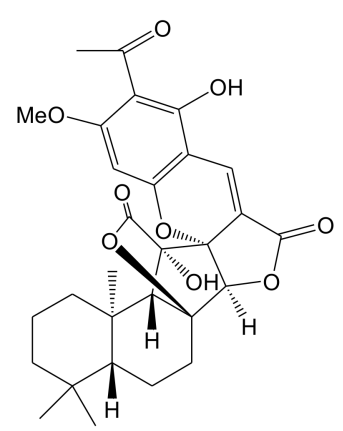

90

Figure 1. The chemical structures of diterpenoids derived from E. fischeriana.

Table 2. Summary of the anti-inflammatory activities of diterpenoids.

\begin{tabular}{|c|c|c|c|c|}
\hline No. & Bioactive Ingredient & Model & Conclusions & Ref. \\
\hline \multirow[t]{2}{*}{1} & \multirow[t]{2}{*}{ jolkinolide B } & $\begin{array}{l}\text { LPS-induced ALI } \\
\text { mouse model }\end{array}$ & $\begin{array}{l}\text { it has a protective effect on } \\
\text { LPS-induced ALI in mice, the } \\
\text { anti-inflammatory mechanism of JB } \\
\text { may be attributed to its suppression } \\
\text { of NF- } \kappa B \text { and MAPK activation }\end{array}$ & [29] \\
\hline & & $\begin{array}{l}\text { LPS-induced RAW } \\
264.7 \text { macrophages }\end{array}$ & $\begin{array}{l}\text { it exhibited inhibitory effect on } \mathrm{NO} \\
\text { production }\left(\mathrm{IC}_{50} 4.9 \mu \mathrm{M}\right)\end{array}$ & [8] \\
\hline 5 & 17-hydroxyjolkinolide B & $\begin{array}{l}\text { LPS stimulated } \\
\text { RAW264 murine } \\
\text { macrophages }\end{array}$ & $\begin{array}{l}\text { it can inhibit inflammatory mediators } \\
\text { but activate heme oxygenase- } 1 \\
\text { expression in LPS-stimulated murine } \\
\text { macrophages }\end{array}$ & [30] \\
\hline 8 & $\begin{array}{l}\text { 11ß-hydroxy-8,14-epoxy-ent- } \\
\text { abieta-13(15)-en-16,12 } \alpha \text {-olide }\end{array}$ & $\begin{array}{l}\text { LPS-induced RAW } \\
264.7 \text { macrophages }\end{array}$ & $\begin{array}{l}\text { it exhibited inhibitory effect on } \mathrm{NO} \\
\text { production }\left(\mathrm{IC}_{50} 12.6 \mu \mathrm{M}\right)\end{array}$ & [8] \\
\hline 23 & yuexiandajisu D & $\begin{array}{l}\text { LPS-induced RAW } \\
264.7 \text { macrophages }\end{array}$ & $\begin{array}{l}\text { it exhibited inhibitory effect on } \mathrm{NO} \\
\text { production }\left(\mathrm{IC}_{50} 5.6 \mu \mathrm{M}\right)\end{array}$ & [8] \\
\hline 52 & ebractenoid F & $\begin{array}{l}\text { LPS-induced RAW } \\
264.7 \text { macrophages }\end{array}$ & $\begin{array}{l}\text { it exhibited inhibitory effect on } \mathrm{NO} \\
\text { production }\left(\mathrm{IC}_{50} 7.4 \mu \mathrm{M}\right)\end{array}$ & [8] \\
\hline 88 & jolkinol A & $\begin{array}{l}\text { LPS-induced RAW } \\
264.7 \text { macrophages }\end{array}$ & $\begin{array}{l}\text { it exhibited inhibitory effect on } \mathrm{NO} \\
\text { production }\left(\mathrm{IC}_{50} 9.4 \mu \mathrm{M}\right)\end{array}$ & [8] \\
\hline
\end{tabular}

\subsection{Anti-HIV-1}

Prostratin (28), a tigliane-type diterpenoid, has been shown to be highly effective in inducing HIV-1 reactivation in latent reservoirs of infected Jurkat-LAT-GFP cells [5,31]. Five tigliane-type diterpenoids, including prostratin (28), fischeroside A (37), fischeroside B (38), fischeroside C (39), 12-deoxyphorbol-13,20-diacetate (40), were tested for cytotoxicities against C8166 cells. Compounds 37-39 showed weak activity in preventing the cytopathic effects of HIV-1 in C8166 cells [12]. Prostratin exerted the strongest anti-HIV-1 activity, with an $\mathrm{EC}_{50}$ of $0.00006 \mu \mathrm{M}$ and a TI of 8500 [12]. 12-Deoxyphorbol-13,20-diacetate displayed anti-HIV-1 activity, with an $\mathrm{EC}_{50}$ of $0.003 \mu \mathrm{M}$ and a TI of 366.67 [12]. This assay demonstrated that introducing an O-acetyl or glucopyranosyl moiety at C-20 of prostratin may dramatically reduce its anti-HIV-1 activity [12]. 


\subsection{Feeding Deterrent}

Feeding deterrent activities of 17-hydroxyjolkinolide A (3), 17-hydroxyjolkinolide B (5), jolkinolide B (1), 12-deoxyphorbol 13-(9Z)-octadecenoate 20-acetate (34) have been studied against two stored-product insects, T. castaneum and S. zeamais [4]. The experiment results are shown in Table 3. Jolkinolide B exhibited significant feeding deterrent activity against $S$. zeamais $\left(\mathrm{EC}_{50}=342.1\right)$ and T. castaneum adults $\left(\mathrm{EC}_{50}=361.4\right)[4]$.

Table 3. Feeding deterrent activities of diterpenoids from Euphorbia fischeriana.

\begin{tabular}{ccccc}
\hline No. & Compound & Insect Type & EC $_{\mathbf{5 0}}(\mathbf{p p m})$ & Ref. \\
\hline \multirow{2}{*}{1} & jolkinolide B & S. Zeamais & 342.1 & \\
& & T. castaneum & 361.4 & \\
3 & 17-hydroxyjolkinolide B & S. Zeamais & 543.9 & {$[4]$} \\
& & T. castaneum & 551.5 & \\
5 & 17-hydroxyjolkinolide A & S. Zeamais & 631.9 \\
& 12-deoxyphorbol & T. castaneum & 656.5 \\
35 & S. Zeamais & 884.3 \\
& 13-(9Z)-octadecenoate 20-acetate & T. castaneum & 1058.4 \\
\hline
\end{tabular}

\subsection{Other Activities}

Three diterpenoids $4 \beta, 9 \alpha$,20-trihydroxy-13,15-secotiglia-1,6-diene-3,13-dione 20-O- $\beta$-D-[6-galloyl]glucopyranoside (45), euphopiloside A (46), ent-8(14)-pimarene-12 $\beta, 15 S, 16$-triol (87) displayed moderate inhibitory effects against $\alpha$-glucosidase [13]. These compounds could be applied to slow down the glucose level for diabetes [13]. 17-Hydroxyjolkinolide B (3) exhibited an inhibitory effect against mycobacterium smegmatis [1]. This means that it possesses a potential antituberculosis effect [1]. 17-Hydroxy-jolkinolide A (5) exerted an inhibitory effect on bone loss by preventing osteoclast formation and bone resorption [32]. 17-Hydroxy-jolkinolide A (5) treatment led to down-regulation of the expression of tartrate-resistant acid phosphatase (TRAP), cathepsin K (Cts K) and MMP-9 [32]. This compound may be useful as a therapeutic reagent for bone loss-associated diseases [32]. Here, we summarize these studies in Table 4.

Table 4. Other bioactive diterpenoids from Euphorbia fischeriana.

\begin{tabular}{|c|c|c|c|}
\hline No. & Bioactive Ingredient & Pharmacological Activity & Ref. \\
\hline 3 & 17-hydroxyjolkinolide B & $\begin{array}{l}\text { antituberculosis effect (it exhibited the inhibitory } \\
\text { effect against mycobacterium smegmatis) }\end{array}$ & [1] \\
\hline 5 & 17-hydroxyjolkinolide A & $\begin{array}{l}\text { anti-osteoporosis (it can prevent osteoclast } \\
\text { formation and bone resorption) }\end{array}$ & [32] \\
\hline 45 & $\begin{array}{l}4 \beta, 9 \alpha, 20 \text {-trihydroxy-13,15-secotiglia- } \\
\text { 1,6-diene-3,13-dione } 20-O-\beta \text {-D- } \\
\text { [6-galloyl]glu-copyranoside }\end{array}$ & $\begin{array}{l}\text { anti-diabetic effect (it possesses the moderate } \\
\text { inhibitory effects against } \alpha \text {-glucosidase) }\end{array}$ & [13] \\
\hline 46 & euphopiloside A & $\begin{array}{l}\text { anti-diabetic effect (it possesses the moderate } \\
\text { inhibitory effects against } \alpha \text {-glucosidase) }\end{array}$ & [13] \\
\hline 87 & ent-8(14)-pimarene-12ß,15S,16-triol & $\begin{array}{l}\text { anti-diabetic effect (it possesses the moderate } \\
\text { inhibitory effects against } \alpha \text {-glucosidase) }\end{array}$ & [13] \\
\hline
\end{tabular}

\section{Conclusions}

Natural plants contain a variety of bioactive compounds. They are frequently used as drugs or lead compounds in drug development. Almost $70 \%$ of modern drugs have a natural product origin $[5,33,34]$. Therefore, searching for compounds with important biological activities from natural plants is of great significance. Diterpenoids are a constant focus of drug discovery because of their great structural diversity, resulting in various bioactivities. E. fischeriana is especially rich in diterpenoids [34]. In this 
paper, we have summarized 90 diterpenoids that have been isolated and identified from E. fischeriana, many of which are novel diterpenoids. Up to now, the majority of these diterpenoids have not been studied in terms of their biological activities. We hope to discover more potential drug leads in the future study. The mechanisms of bioactive ingredients from E. fischeriana need deeper research. Thoroughly understanding the targets for active compounds will help us to design effective new drugs. This review provides reference for further research of these diterpenoids and lays the foundation for drug discovery.

Acknowledgments: This work was supported by a grant from the National Natural Science Foundation of China (No. 81573660) and the Intramural Research Program of the Qiqihar Medical University (No. QY2016Z-01).

Author Contributions: Baiyu Jian conceived and wrote the manuscript; Hao Zhang collected the literature; Jicheng Liu revised the manuscript.

Conflicts of Interest: The authors declare no conflict of interest

\section{References}

1. Wang, C.J.; Yan, Q.L.; Ma, Y.F.; Sun, C.P.; Chen, C.M.; Tian, X.G.; Han, X.Y.; Wang, C.; Deng, S.; Ma, X.C. Ent-Abietane and Tigliane Diterpenoids from the Roots of Euphorbia fischeriana and Their Inhibitory Effects against Mycobacterium Smegmatis. J. Nat. Prod. 2017, 80, 1248-1254. [CrossRef] [PubMed]

2. Wang, H.B.; Chen, W.; Zhang, Y.Y.; Wang, X.Y.; Liu, L.P.; Tong, L.J.; Chen, Y. Four New Diterpenoids from the Roots of Euphorbia fischeriana. Fitoterapia 2013, 91, 211-216. [CrossRef] [PubMed]

3. Sun, Y.X.; Liu, J.C. Chemical Constituents and Biological Activities of Euphorbia fischeriana Steud. Chem. Biodivers. 2011, 8, 1205-1214. [CrossRef] [PubMed]

4. $\quad$ Geng, Z.F.; Liu, Z.L.; Wang, C.F.; Liu, Q.Z.; Shen, S.M.; Liu, Z.M.; Du, S.S.; Deng, Z.W. Feeding Deterrents against Two Grain Storage Insects from Euphorbia fischeriana. Molecules 2011, 16, 466-476. [CrossRef] [PubMed]

5. Vasas, A.; Hohmann, J. Euphorbia Diterpenes: Isolation, Structure, Biological Activity, and Synthesis (2008-2012). Chem. Rev. 2014, 114, 8579-8612. [CrossRef] [PubMed]

6. Wang, Y.B.; Huang, R.; Wang, H.B.; Jin, H.Z.; Lou, L.G.; Qin, G.W. Diterpenoids from the Roots of Euphorbia fischeriana. J. Nat. Prod. 2006, 69, 967-970. [CrossRef] [PubMed]

7. Kuang, X.; Li, W.; Kanno, Y.; Yamashita, N.; Kikkawa, S.; Azumaya, I.; Nemoto, K.; Asada, Y.; Koike, K. Euphorins A-H: Bioactive Diterpenoids from Euphorbia fischeriana. J. Nat. Med. 2016, 70, 412-422. [CrossRef] [PubMed]

8. Lee, J.W.; Lee, C.; Jin, Q.; Jang, H.; Lee, D.; Lee, H.J.; Shin, J.W.; Han, S.B.; Hong, J.T.; Kim, Y.; et al. Diterpenoids from the Roots of Euphorbia fischeriana with Inhibitory Effects on Nitric Oxide Production. J. Nat. Prod. 2016, 79, 126-131. [CrossRef] [PubMed]

9. Shi, H.M.; Williams, I.D.; Sung, H.H.; Zhu, H.X.; Ip, N.Y.; Min, Z.D. Cytotoxic Diterpenoids from the Roots of Euphorbia Ebracteolata. Planta Med. 2005, 71, 349-354. [CrossRef] [PubMed]

10. Wang, H.B.; Chu, W.J.; Wang, Y.; Ji, P.; Wang, Y.B.; Yu, Q.; Qin, G.W. Diterpenoids from the Roots of Euphorbia fischeriana. J. Asian Nat. Prod. Res. 2010, 12, 1038-1043. [CrossRef] [PubMed]

11. Wang, M.; Wei, Q.; Wang, Q.H.; Xia, Y.G.; Yang, B.Y.; Kuang, H.X. Antitumor Activity of Tigliane Type Diterpene from Euphorbia fischeriana Steud. Acta Chin. Med. Pharmacol. 2013, 4, 11-14.

12. Pan, L.L.; Fang, P.L.; Zhang, X.J.; Ni, W.; Li, L.; Yang, L.M.; Chen, C.X.; Zheng, Y.T.; Li, C.T.; Hao, X.J.; et al. Tigliane-Type Diterpenoid Glycosides from Euphorbia fischeriana. J. Nat. Prod. 2011, 74, 1508-1512. [CrossRef] [PubMed]

13. Wei, Y.L.; Yu, Z.L.; Huo, X.K.; Tian, X.G.; Feng, L.; Huang, S.S.; Deng, S.; Ma, X.C.; Jia, J.M.; Wang, C. Diterpenoids from the Roots of Euphorbia fischeriana and Their Inhibitory Effects on Alpha-Glucosidase. J. Asian Nat. Prod. Res. 2017, 1-8. [CrossRef]

14. Shi, Q.; Sun, Y.W.; Meng, D. Phytochemical and Cytotoxic Studies on the Roots of Euphorbia fischeriana. Bioorg. Med. Chem. Lett. 2017, 27, 266-270. [CrossRef] [PubMed] 
15. Wang, M.; Wang, Q.; Wei, Q.; Li, J.; Guo, C.; Yang, B.; Kuang, H. Two New ent-Atisanes from the Root of Euphorbia fischeriana Steud. Nat. Prod. Res. 2016, 30, 144-149. [CrossRef] [PubMed]

16. Kuang, X.; Li, W.; Kanno, Y.; Yamashita, N.; Nemoto, K.; Asada, Y.; Koike, K. ent-Atisane Diterpenoids from Euphorbia fischeriana Inhibit Mammosphere Formation in MCF-7 Cells. J. Nat. Med. 2016, 70, 120-126. [CrossRef] [PubMed]

17. Wang, J.L.; Wang, Y.Q.; Li, T.; Wang, H.; Zhao, M.; Zhang, S.J. Study on the Chemical Constituents from Fresh Roots of Euphorbia fischeriana. Zhong Yao Cai 2010, 33, 1406-1409. [PubMed]

18. Wang, M.; Li, J.; Qing, W.; Wang, Q.H.; Yang, B.Y.; Kuang, H.X. Antitumor Activity of Abietane Type Diterpene from Euphorbia fischeriana Steud. China Pharm. 2015, 8, 1261-1262.

19. Xu, H.Y.; Chen, Z.W.; Hou, J.C.; Du, F.X.; Liu, J.C. Jolkinolide B Induces Apoptosis in MCF-7 Cells through Inhibition of the PI3k/Akt/Mtor Signaling Pathway. Oncol. Rep. 2013, 29, 212-218. [CrossRef] [PubMed]

20. Lin, Y.; Cui, H.; Xu, H.; Yue, L.; Xu, H.; Jiang, L.; Liu, J. Jolkinolide B Induces Apoptosis in MDA-MB-231 Cells through Inhibition of the PI3k/Akt Signaling Pathway. Oncol. Rep. 2012, 27, 1976-1980. [PubMed]

21. Wang, J.H.; Zhou, Y.J.; Bai, X.; He, P. Jolkinolide B from Euphorbia fischeriana Steud Induces Apoptosis in Human Leukemic U937 Cells through PI3k/Akt and Xiap Pathways. Mol. Cells 2011, 32, 451-457. [CrossRef] [PubMed]

22. Luo, H.; Wang, A. Induction of Apoptosis in K562 Cells by Jolkinolide B. Can. J. Physiol. Pharmacol. 2006, 84, 959-965. [CrossRef] [PubMed]

23. Sun, C.; Cui, H.; Yang, H.; Du, X.; Yue, L.; Liu, J.; Lin, Y.U. Anti-Metastatic Effect of Jolkinolide B and the Mechanism of Activity in Breast Cancer Mda-Mb-231 Cells. Oncol. Lett. 2015, 10, 1117-1122. [CrossRef] [PubMed]

24. Yan, S.S.; Li, Y.; Wang, Y.; Shen, S.S.; Gu, Y.; Wang, H.B.; Qin, G.W.; Yu, Q. 17-Acetoxyjolkinolide B Irreversibly Inhibits Ikappab Kinase and Induces Apoptosis of Tumor Cells. Mol. Cancer Ther. 2008, 7, 1523-1532. [CrossRef] [PubMed]

25. Wang, Y.; Ma, X.; Yan, S.; Shen, S.; Zhu, H.; Gu, Y.; Wang, H.; Qin, G.; Yu, Q. 17-Hydroxy-Jolkinolide B Inhibits Signal Transducers and Activators of Transcription 3 Signaling by Covalently Cross-Linking Janus Kinases and Induces Apoptosis of Human Cancer Cells. Cancer Res. 2009, 69, 7302-7310. [CrossRef] [PubMed]

26. Xu, H.Y.; Chen, Z.W.; Li, H.; Zhou, L.; Liu, F.; Lv, Y.Y.; Liu, J.C. 12-Deoxyphorbol 13-Palmitate Mediated Cell Growth Inhibition, G2-M Cell Cycle Arrest and Apoptosis in BGC823 Cells. Eur. J. Pharmacol. 2013, 700, 13-22. [CrossRef] [PubMed]

27. Xu, H.Y.; Pan, Y.M.; Chen, Z.W.; Lin, Y.; Wang, L.H.; Chen, Y.H.; Jie, T.T.; Lu, Y.Y.; Liu, J.C. 12-Deoxyphorbol 13-Palmitate Inhibit Vegf-Induced Angiogenesis Via Suppression of Vegfr-2-Signaling Pathway. J. Ethnopharmacol. 2013, 146, 724-733. [CrossRef] [PubMed]

28. Jian, B.; Zhang, H.; Han, C.; Liu, J. Anti-Cancer Activities of Diterpenoids Derived from Euphorbia fischeriana Steud. Molecules 2018, 23, 387. [CrossRef] [PubMed]

29. Yang, H.; Li, Y.; Huo, P.; Li, X.O.; Kong, D.; Mu, W.; Fang, W.; Li, L.; Liu, N.; Fang, L.; et al. Protective Effect of Jolkinolide B on Lps-Induced Mouse Acute Lung Injury. Int. Immunopharmacol. 2015, 26, 119-124. [CrossRef] [PubMed]

30. Uto, T.; Qin, G.W.; Morinaga, O.; Shoyama, Y. 17-Hydroxy-Jolkinolide B, a Diterpenoid from Euphorbia fischeriana, Inhibits Inflammatory Mediators but Activates Heme Oxygenase-1 Expression in Lipopolysaccharide-Stimulated Murine Macrophages. Int. Immunopharmacol. 2012, 12, 101-109. [CrossRef] [PubMed]

31. Warrilow, D.; Gardner, J.; Darnell, G.A.; Suhrbier, A.; Harrich, D. Hiv Type 1 Inhibition by Protein Kinase C Modulatory Compounds. AIDS Res. Hum. Retrovir. 2006, 22, 854-864. [CrossRef] [PubMed]

32. Wang, Y.; Xu, X.; Wang, H.B.; Wu, D.; Li, X.O.; Peng, Q.; Liu, N.; Sun, W.C. 17-Hydroxy-Jolkinolide a Inhibits Osteoclast Differentiation through Suppressing the Activation of Nf-Kappab and Mapks. Int. Immunopharmacol. 2015, 29, 513-520. [CrossRef] [PubMed] 
33. Newman, D.J.; Cragg, G.M. Natural Products as Sources of New Drugs over the 30 Years from 1981 to 2010. J. Nat. Prod. 2012, 75, 311-335. [CrossRef] [PubMed]

34. Ahmed, A.A.; Mahmoud, A.A.; Ahmed, U.M.; El-Bassuony, A.A.; El-Razk, M.H.A.; Pare, P.W.; Karchesy, J. Manoyl Oxide Alpha-Arabinopyranoside and Grindelic Acid Diterpenoids from Grindelia Integrifolia. J. Nat. Prod. 2001, 64, 1365-1367. [CrossRef] [PubMed]

(C) 2018 by the authors. Licensee MDPI, Basel, Switzerland. This article is an open access article distributed under the terms and conditions of the Creative Commons Attribution (CC BY) license (http:// creativecommons.org/licenses/by/4.0/). 